\title{
Éditorial/Commentary
}

\section{La médecine d'urgence et l'administration médicale: partir à la découverte de ce carrefour}

\author{
James Ducharme, MD
}

Le médecin n'apprend pas tout ce qu'il doit savoir et maîtriser seulement dans les grands collèges... Aussi dois-tu étudier jour après jour sans relâche, examiner et observer avec diligence; ne méprise rien, et ne te surestime pas avec désinvolture... Apprends aussi de ceux qui ont plus d'expérience que toi, car qui peut prétendre tout savoir?

- Paracelse

Dans le présent numéro, le fournal canadien de médecine d'urgence ( $\mathcal{F C M U}$ ) est fier de lancer sa nouvelle série d'articles thématiques. Au cours des 2 dernières années, sous la direction de Jonathan Sherbino, le $7 C M U$ a publié une série d'articles sur l'enseignement médical. Ces articles ont suscité un enthousiasme tel que nous avons décidé de publier une série semblable d'articles sélectionnés sur l'administration médicale. Et qui de mieux placé que Grant Innes, rédacteur en chef émérite du $7 C M U$, pour diriger cette série? On a demandé à M. Innes de trouver tous les sujets de la série, d'inviter des experts en la matière à participer au projet, et de superviser la mise au point de tous les articles soumis. Aussi le premier article de cette série, intitulé « Your Profession Needs You (Lessons in Medical Leadership) $\gg,{ }^{1}$ convient-il parfaitement. L'article a été rédigé par deux chefs de file chevronnés en administration de la médecine d'urgence au Canada, soit Tim Rutledge et Doug Sinclair; tous deux occupent maintenant des postes de haute direction dans leur hôpital respectif.

L'ADN des médecins d'urgence permet la survie et le développement dans un environnement stressant et bourdonnant d'activité, là où les décisions pour sauver des vies se prennent en quelques secondes; il semblerait donc que la lourdeur apparente de l'administration hospitalière entre en parfaite contradiction avec leur
ADN. Pourtant, MM. Rutledge et Sinclair font remarquer à juste titre que le sens de la conduite fait aussi partie de l'ADN des médecins d'urgence, puisqu'ils doivent diriger des équipes tous les jours au chevet des malades. Ils font également une distinction très nette entre « diriger » et « gérer ». Ce dernier terme implique une attention particulière portée aux détails, la surveillance des principaux indicateurs de performance, et une évolution de la situation en cours par la résolution de conflits et la mise en oeuvre de politiques et de procédures; tandis que le premier terme exige une ouverture sur le monde extérieur, du charisme, une pensée stratégique, et la capacité de rallier diverses opinions pour finalement en arriver à une approche commune.

Certains diront qu'on naît chef, qu'on ne le devient pas; et je suis d'accord en grande partie avec eux sur ce point. La capacité innée de diriger ne mène nulle part sans une construction mentale appropriée; cependant, cela s'apprend par la formation et l'expérience. Le sens de la conduite est fondamental; nombreux sont ceux qui pensent que la médecine d'urgence a fait preuve d'absence de direction depuis trop longtemps, dans trop de circonstances. Bon nombre de nos meilleurs chefs continuent à exercer leur aptitude à diriger en dehors des services d'urgence (SU); les deux auteurs de l'article publié dans le présent numéro sont d'excellents exemples d'une telle croissance professionnelle. Nous devons toutefois veiller à ce que nos chefs, tant à l'intérieur qu'à l'extérieur des SU au Canada, continuent à faire progresser la médecine d'urgence. L'expérience bâtie au fil des ans par les interactions établies à l'extérieur des services d'urgence très fréquentés ne peut qu'améliorer davantage l'administration de ces services.

Tiré de Department of Emergency Medicine, McMaster University, Hamilton, ON.

Correspondence to: Dr. James Ducharme, 6711 Mississauga Road, Suite 404, Mississauga, ON L5N 2W3; paindoc22000@yahoo.com.

Cet article a été évalué par des pairs.

(c) Canadian Association of Emergency Physicians

DOI 10.2310/8000.2013.131125F 
Les chefs ont une responsabilité envers ceux qui leur font confiance, en l'occurrence les médecins d'urgence. Comme le disait Henry Kissinger: « Les chefs sont responsables non pas des sondages d'opinion mais des conséquences de leurs actions $\gg$. Le sens de la conduite exige une bonne dose de conviction et de confiance en soi parce que « si vous désirez propulser votre organisation sur le devant de la scène, vous devez susciter les divergences d'opinion, les désaccords et la vérité parmi vos associés, et vous-même vous devez aller à la recherche de la vérité $\gg .{ }^{2}$ Être chef ne signifie pas que vous avez la science infuse ou que vous avez raison; cela signifie que vous devez être capable de mener chacun là où vous le souhaitez. Cela signifie également que vous devez, en tant que chef, être plus disposé que tout autre à admettre vos torts.

Aussi le $7 C M U$ a-t-il demandé à Grant Innes d'incarner ce genre de chef et de nous faire découvrir la complexité de l'administration médicale par un choix approprié de sujets et de collaborateurs, experts en la matière. Forts de ces nouvelles connaissances, nous serons tous en mesure de mieux franchir les étapes nécessaires pour améliorer les soins dans nos hôpitaux respectifs ou pour communiquer avec nos pairs au cours de réunions de comité. Cela nous donnera également des munitions pour plaider plus efficacement en faveur des patients et de la médecine d'urgence elle-même. J'espère que cette nouvelle série vous plaira autant que je le pressens moi-même.

Intérêts concurrentiels: aucun intérêt déclaré.

\section{RÉFÉRENCES}

1. Rutledge T, Sinclair D. Your profession needs you (lessons in medical leadership). CFEM 2013;15:00-00.

2. Bennis W. Why leaders can't lead. San Francisco: Jossey-Bass; 1989. 